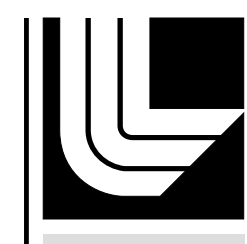

LA W REN CE LIVERM ORE N A TIO NAL LABORATORY

\title{
FIRST-PRINCIPLES PHASE STABILITY IN THE TI-V ALLOY SYSTEM
}

P. Soderlind, A. Landa, L. H. Yang, A.

Teweldebrhan

April 17, 2013

Journal of Alloys and Compounds 
This document was prepared as an account of work sponsored by an agency of the United States government. Neither the United States government nor Lawrence Livermore National Security, LLC, nor any of their employees makes any warranty, expressed or implied, or assumes any legal liability or responsibility for the accuracy, completeness, or usefulness of any information, apparatus, product, or process disclosed, or represents that its use would not infringe privately owned rights. Reference herein to any specific commercial product, process, or service by trade name, trademark, manufacturer, or otherwise does not necessarily constitute or imply its endorsement, recommendation, or favoring by the United States government or Lawrence Livermore National Security, LLC. The views and opinions of authors expressed herein do not necessarily state or reflect those of the United States government or Lawrence Livermore National Security, LLC, and shall not be used for advertising or product endorsement purposes. 


\title{
First-principles phase stability in the Ti-V alloy system
}

Per Söderlind, Alex Landa, Lin H. Yang, and Amanuel M. Teweldeberhan Condensed Matter and Materials Division, Lawrence Livermore National Laboratory, Livermore CA 94550, USA

\begin{abstract}
Phase stability in the transition metals are mostly dictated by the bonding of the $d$ electrons and is believed to be fairly well understood from either a canonical-band picture or a tight-binding model. These models are related and capture the fact that as one proceeds through the nonmagnetic $d$-transition series one encounters the hexagonal closepacked (hcp), body-centered cubic (bcc), hcp, and face-centered cubic (fcc) phases. This structural sequence depends on the gradual filling of the $d$ band (roughly one electron per atomic number increase) that is altered when magnetism is present simply due to the spin polarization of the $d$ band. However, recent more careful experimental and theoretical studies have shown that the aforementioned appealing models do not entirely explain the bonding and phase stability in the transition metals. First, there is a destabilization of the bcc phase due to pressure in the Group $\mathrm{Vb}$ metals ( $\mathrm{V}, \mathrm{Nb}$, and $\mathrm{Ta}$ ) and second, a temperature-induced stabilization of the bcc phase in the Group IVb (Ti, Zr, and Hf) metals even though at low temperatures it is strongly unstable. Here we address the latter phenomenon and study the influence of alloying titanium with its next neighbor vanadium by applying the recently developed self-consistent $a b$ initio lattice dynamics (SCAILD) approach that has heretofore never been utilized for an alloy system.
\end{abstract}

KEYWORDS: transition metals, phase stability, alloys, phonons, density-functional theory 


\section{Introduction}

Tremendous strides have been made to understand the electronic structure, bonding, and phase stability of many metals in the Periodic Table since the conception of densityfunctional theory (DFT) almost half a decade ago [1,2]. Skriver [3] showed that the ground state of most metals is correctly reproduced from DFT electronic structures combined with the atomic-sphere approximation for the crystal geometries. Focusing on the $d$-transition metals, it was explained that the structural sequence hcp, bcc, hcp, and fcc was due to a gradual filling of the $d$ band for these metals with little disturbance from other electron states. The canonical-band picture [3] used for this purpose is actually equivalent to the tight-binding form Duthie and Pettifor applied to explain the groundstate phases of the early rare-earth metals [4]. The early successes of these models depend on the fact that the structures in the transition and rare-earth metals are dictated by $d$-electron states that dominate the bonding.

More recently is has become apparent that neither of these elegant but somewhat simplistic models is sufficient to explain phase stability in the transition metals. A series of papers [5-10] report, for instance, the existence of a rhombohedral phase (contrary to cubic or hexagonal) in vanadium in the 60-70 GPa pressure range. Here, delicate details of the electronic structure (nesting) close to the Fermi level, emphasized during compression, are essential for the bcc-structure destabilization and similar effects have also been found in $\mathrm{Nb}$ and $\mathrm{Ta}[9,10]$. Next, the high-temperature stabilization of the bcc phase in the Group IVb metals ( $\mathrm{Ti}, \mathrm{Zr}$, and $\mathrm{Hf}$ ) cannot be explained from the ground-state electronic structure obtained from DFT. In this latter case the DFT electronic structure actually predicts mechanical instability of the bcc phase at lower temperatures [11].

In the present report we are addressing the latter phenomenon and particularly the influence of alloying on the stability field of the bec phase in titanium (Ti). For this purpose we apply a recently developed scheme to take phonon-phonon interaction into account at elevated temperatures within the self-consistent $a b$ initio lattice dynamics (SCAILD) method [12]. SCAILD has addressed elemental metals but never before alloy systems. The approach is described in great detail [13] and not repeated here, but the general idea is to account for thermal vibrations of the atoms and their interactions. This is accomplished by (i): calculating the forces on atoms, displaced from the ideal bcc 
positions according to the temperature, and (ii): computing the phonon density of states (DOS). Because the forces, displacements, and the phonon DOS depend on each other the scheme is set up to self-consistently determine the temperature dependence of the lattice dynamics. If the procedure converges and the bcc entropy is large enough at the studied temperature bcc stabilization will occur.

Section 2 deals with computational parameters and methods for the densityfunctional theory model that is used for the force calculations. In section 3 we present and discuss our results for the Ti-V alloy system, and conclude with a discussion in section 4 .

\section{Computational Details}

All calculations are performed within the framework of DFT and the necessary assumption for the unknown electron exchange and correlation functional is that of the local density approximation (LDA) [14]. Although newer varieties of this approximation have been proposed, our test calculations reveal that the calculated forces are easier to converge with LDA compared to other formulations. Our particular implementation is based on the full-potential linear muffin-tin orbitals (FPLMTO) method that has recently been reviewed [15]. The basis functions are well converged, specifically, we associate a set of semi-core states $3 s$ and $3 p$ and valence states $4 s, 4 p$, and $3 d$ to two kinetic energy parameters for a so-called double basis set. For the SCAILD simulation a bec super cell is required and similar to previous investigations [12] we are applying a 4x4x4 (64 atom) cell size. In all present calculations the sampling of $k$ points in the Brillouin zone (BZ) for the appropriate summations are carefully checked for convergence and $64 \mathrm{k}$ points are sampled in the irreducible part of the BZ.

In order to study the Ti-V alloy system we conveniently apply the virtual crystal approximation (VCA) that is usually applicable when alloying metals that are neighbors in the Periodic Table. The concept of the VCA is to replace the true alloy with an artificial metal that consists of a weighted average between the two components. For example, the $\mathrm{VCA} \mathrm{Ti}_{80} \mathrm{~V}_{20}$ alloy is simply a monoatomic metal with a nuclear charge of 22.2 and a total of 22.2 electrons. The VCA has been shown to effectively model the behavior of the elastic constants for the $d$-transition alloys [16] and this lends credence to its utilization here for the Ti-V alloy system. 


\section{Stability of the Ti-V alloy system}

Let us first center our attention on titanium metal. In Fig. 1 we show the experimental phase diagram for titanium [17] at ambient pressure and it undergoes a phase transition to the bcc phase close to $1165 \mathrm{~K}\left(892^{\circ} \mathrm{C}\right)$ from which it ultimately melts. Previous SCAILD calculations [12] explain this (hcp to bcc) phase transformation to be driven by entropy contributions to the free energies associated with phonon-phonon interactions. At lower temperatures, as mentioned in the introduction, the bcc phase of $\mathrm{Ti}$ is not even mechanically stable which obviously is a necessary but not sufficient condition for its existence. One can thus define a mechanical-stability temperature as a lower bound to the actual phase line and by performing SCAILD simulations for bcc Ti at various temperatures search for the lowest temperature that stabilizes all phonon branches. With this procedure we bracket the stabilization temperature to about $700{ }^{\circ} \mathrm{C}$ for unalloyed $\mathrm{Ti}$ and this is about $200{ }^{\circ} \mathrm{C}$ lower than the phase transitions in Fig. 1 and thus consistent with a lower bound.

In Fig. 2 we show the experimental Ti-V alloy phase at ambient pressure [18]. Notice, on the left (Ti) side the phase line drops very strongly with small amounts of $\mathrm{V}$ in the Ti-V alloy up until about $40 \%$ vanadium. This phase line suggests that stability of the bcc phase of Ti is extremely sensitive to addition of vanadium, which we find intriguing and investigate from first-principles modeling and the SCAILD technique. Rather than calculating the actual phase line for the alloy system, which entails calculating accurate free energies for both phases, we focus on the lower bound defined by mechanical stability, hoping that the driving forces for this stabilization are also responsible for the hep to bec transformation.

We perform SCAILD simulations for bec Ti and two bec Ti-V alloys $\left(\mathrm{Ti}_{80} \mathrm{~V}_{20}\right.$ and $\mathrm{Ti}_{60} \mathrm{~V}_{40}$ ) as a function of temperature in steps of about $50{ }^{\circ} \mathrm{C}$ paying attention to the lowest temperature that stabilizes all phonons. In Fig. 3 we show these phonon frequencies for $\mathrm{Ti}, \mathrm{Ti}_{80} \mathrm{~V}_{20}$, and $\mathrm{Ti}_{60} \mathrm{~V}_{40}$ and find that the $\mathrm{T}_{1}$ in $\Gamma-\mathrm{N}$ and $\mathrm{T}$ in $\Gamma$-P branches differ the most between $\mathrm{Ti}$ and its $\mathrm{Ti}-\mathrm{V}$ alloys. The former is related to the elastic constant C' (see below) and is strongly unstable for Ti at low temperatures, shown in Fig. 3 as a dashed line. Many branches are similar but scale relative to each other. There will 
of course be scaling effects due to the differences in temperatures, atomic masses, and equilibrium volumes between $\mathrm{Ti}$ and the Ti-V alloys.

Next, in Fig. 4 we plot the mechanical-stability temperatures (black squares) together with a part of the experimental phase diagram. The temperature dependence of the stabilization, as a function of alloy composition, appears to closely match the behavior of the phase line. Acknowledging that these temperatures are lower bounds, they are expected to be lying below the phase boundary. When adding a constant $200{ }^{\circ} \mathrm{C}$ offset (red solid circles) in Fig. 4 to account for this, we clearly reproduce the experimental trend. It thus appears likely that the bcc mechanical stabilization is a precursor to the hep to bcc phase transition with similar physical origin.

\section{Discussion}

By applying DFT calculations for the Ti-V alloy system, combined with the selfconsistent lattice dynamics scheme, we find that the bcc phase stabilization has a temperature dependency on composition that is identical to the bcc phase boundary but shifted to lower temperatures. This makes perfect sense because the mechanical stabilization is a lower bound to the transition and their similar behavior advocate that the physical mechanism that dictates the stability of the bcc phase also plays a major role in the shape of the phase line. Because of the negative slope, lower temperatures are required (less entropy) to induce the hep to bcc transformation with increasing vanadium composition, indicating that the bcc instability becomes weaker. In order to interrogate this further we calculate, at zero temperature and at the equilibrium volume, the tetragonal shear elastic constant (C') [16] as a function of Ti-V composition and show the results in Fig. 5. For Ti metal, the instability is relatively strong because $C^{\prime}$ is greatly negative in agreement with the result of a previous theoretical study [19]. However, small amounts of vanadium strongly increase $C^{\prime}$ and reduce the strength of the instability, in accordance with the behavior of the bcc phase line in the Ti-V alloy. Because C' reflects the long-wave length part of the $T_{1}$ in $\Gamma-\mathrm{N}$ branch, a negative $\mathrm{C}^{\prime}$ is consistent with imaginary phonon frequencies for this branch (see Fig. 3) that apparently can be removed with small amounts of vanadium. 
We conclude that the ground-state (zero temperature) electronic structure is sensitively dependent on $\mathrm{V}$ composition in the Ti-V alloy, as is evident from the C' behavior and the $T_{1} \Gamma-\mathrm{N}$ branch, which in turn explains the similar sensitivity of $\mathrm{V}$ content in the phase diagram. Furthermore, the DFT-SCAILD technique seems to perform well not only for elemental metals [12] but also for the Ti-V alloy system.

\section{Acknowledgements}

We thank O. Eriksson, P. Souvatzis, B. Johansson, and B. Grabowski for enriching discussions. Computing support for this work came from the LLNL Computing Grand Challenge program. This work performed under the auspices of the U.S. DOE by LLNL under Contract DE-AC52-07NA27344 and funded by the Laboratory Directed Research and Development Program at LLNL under project tracking code 11-ER-033.

\section{References}

[1] P. Hohenberg and W. Kohn, Phys. Rev. 136, B864 (1964).

[2] W. Kohn and L. Sham, Phys. Rev. 140, A113 (1965).

[3] H. L. Skriver, Phys. Rev. B 31, 1909 (1985).

[4] J. C. Duthie and D. G. Pettifor, Phys. Rev. Lett. 38, 564 (1977).

[5] A. Landa, J. Klepeis, P. Söderlind, I. Naumov, O. Velikokhatnyi, L. Vitos, and A. Ruban, J. Phys.: Condens. Matter 18, 5079 (2006).

[6] Y. Ding, R. Ahuja, J. Shu, P. Chow, W. Luo, and H. -K. Mao, Phys. Rev. Lett. 98, 085502 (2007).

[7] B. Lee, R. E. Rudd, J. E. Klepeis, P. Söderlind, and A. Landa, Phys. Rev. B 75, 180101(R) (2007).

[8] B. Lee, R. E. Rudd, J. E. Klepeis, and R. Becker, Phys. Rev. B 77, 134105 (2008).

[9] A. Landa, P. Söderlind, A. V. Ruban, O. E. Peil, and L. Vitos, Phys. Rev. Lett. 103, 235501 (2009).

[10] A. Landa, P. Söderlind, O. I. Velikokhatnyi, I. I. Naumov, A. V. Ruban, O. E. Peil, L. Vitos, Phys. Rev. B 82, 144114 (2010).

[11] Y. Chen, C. -L. Fu, K. M. Ho, and B. N. Harmon, Phys. Rev. B 31, 6775 (1985). 
[12] P. Souvatzis, O. Eriksson, M. I. Katsnelson, and S. P. Rudin, Phys. Rev. Lett. 100, 095901 (2008).

[13] P. Souvatzis, O. Eriksson, M. I. Katsnelson, and S. P. Rudin, Com. Mat. Sci. 44, 888 (2008).

[14] U. von Barth and L. Hedin, J. Phys. C 5, 1629 (1972).

[15] J. M. Wills, M. Alouani, P. Andersson, A. Delin, O. Eriksson, and O. Grechnev, Full-Potential Electronic Structure Methods (Springer-Verlag, Berlin, 2010).

[16] P. Söderlind, O. Eriksson, J. M. Wills, and A. M. Boring, Phys. Rev. B 48, 5844 (1993).

[17] D. A. Young in Phase Diagram of the Elements (University of California Press, Berkeley, 1991).

[18] D. B. Chernov, V. V. Molokanov, P. B. Budberg, and A. Y. Shinyaev, in Proceedings of $3^{\text {rd }}$ International Conference on Titanium and Titanium Alloys, Vol. 2, eds. J. C. Williams and A. F. Belov (Plenum Press, New York, 1982), p. 1307. [19] K. Persson, M. Ekman, and V. Ozolins, Phys. Rev. B 61, 11221 (2000). 


\section{Figure Captions}

1. Experimental phase diagram of titanium metal [17].

2. Experimental phase diagram for the Ti-V alloy system [18]. The hatched areas display hep and bec stability regions.

3. Calculated phonon dispersions for $\mathrm{Ti}$ at $700{ }^{\circ} \mathrm{C}, \mathrm{Ti}_{80} \mathrm{~V}_{20}$ at $500{ }^{\circ} \mathrm{C}$, and $\mathrm{Ti}_{60} \mathrm{~V}_{40}$ at $300{ }^{\circ} \mathrm{C}$. The dashed line indicates the unstable $\mathrm{T}_{1} \Gamma-\mathrm{N}$ branch of $\mathrm{Ti}$ at $25^{\circ} \mathrm{C}$.

4. Ti-V alloy experimental phase diagram (black line) together with calculated mechanical-stability temperatures (black squares). The red solid circles show these temperatures offset a constant 200 degrees amount.

5. Calculated tetragonal shear elastic constant $\left(C^{\prime}\right)$ as a function of composition in the Ti-V alloy system. Addition of vanadium removes the tetragonal instability of the bec Ti-V alloy. 
Figure 1

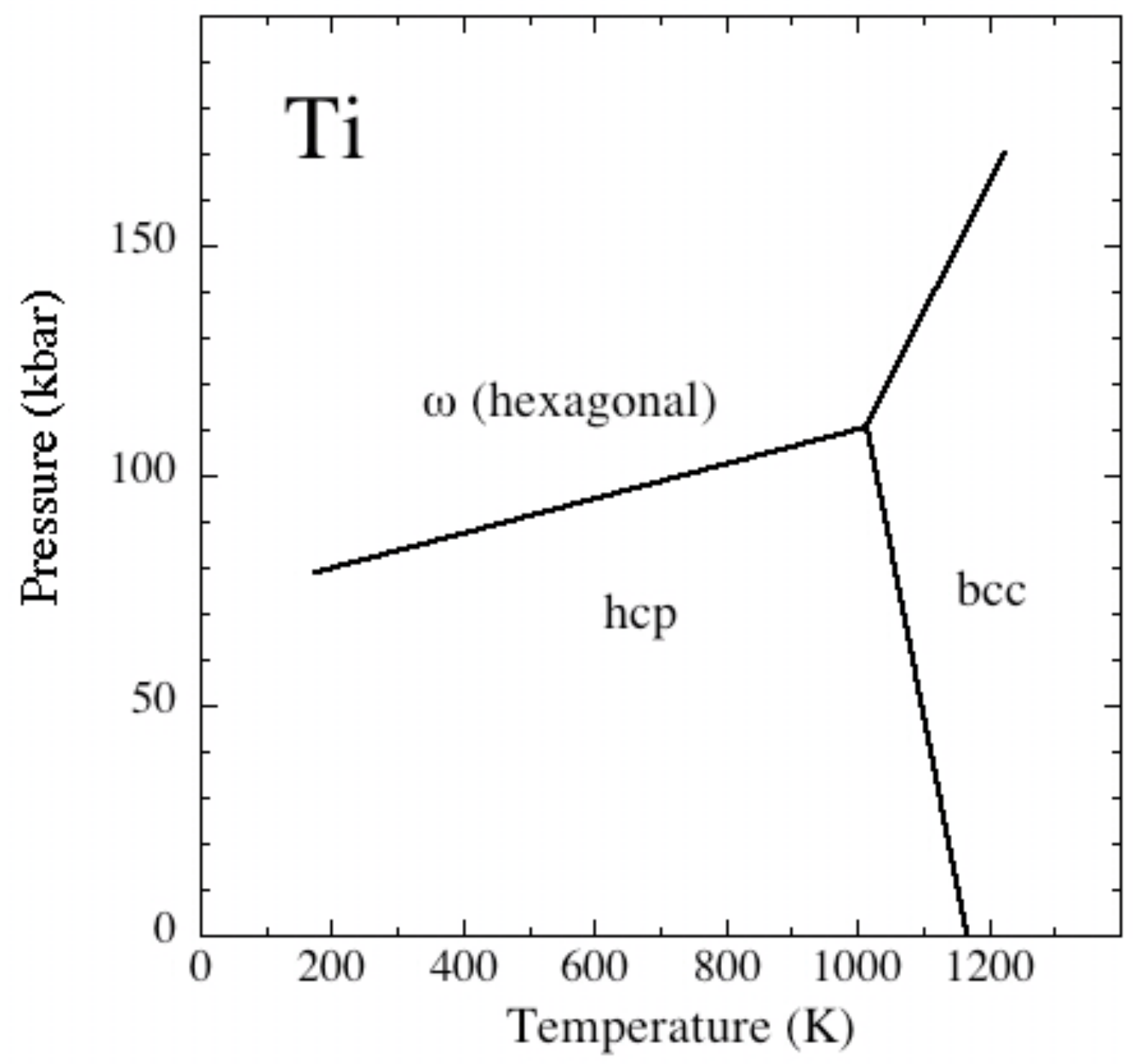


Figure 2

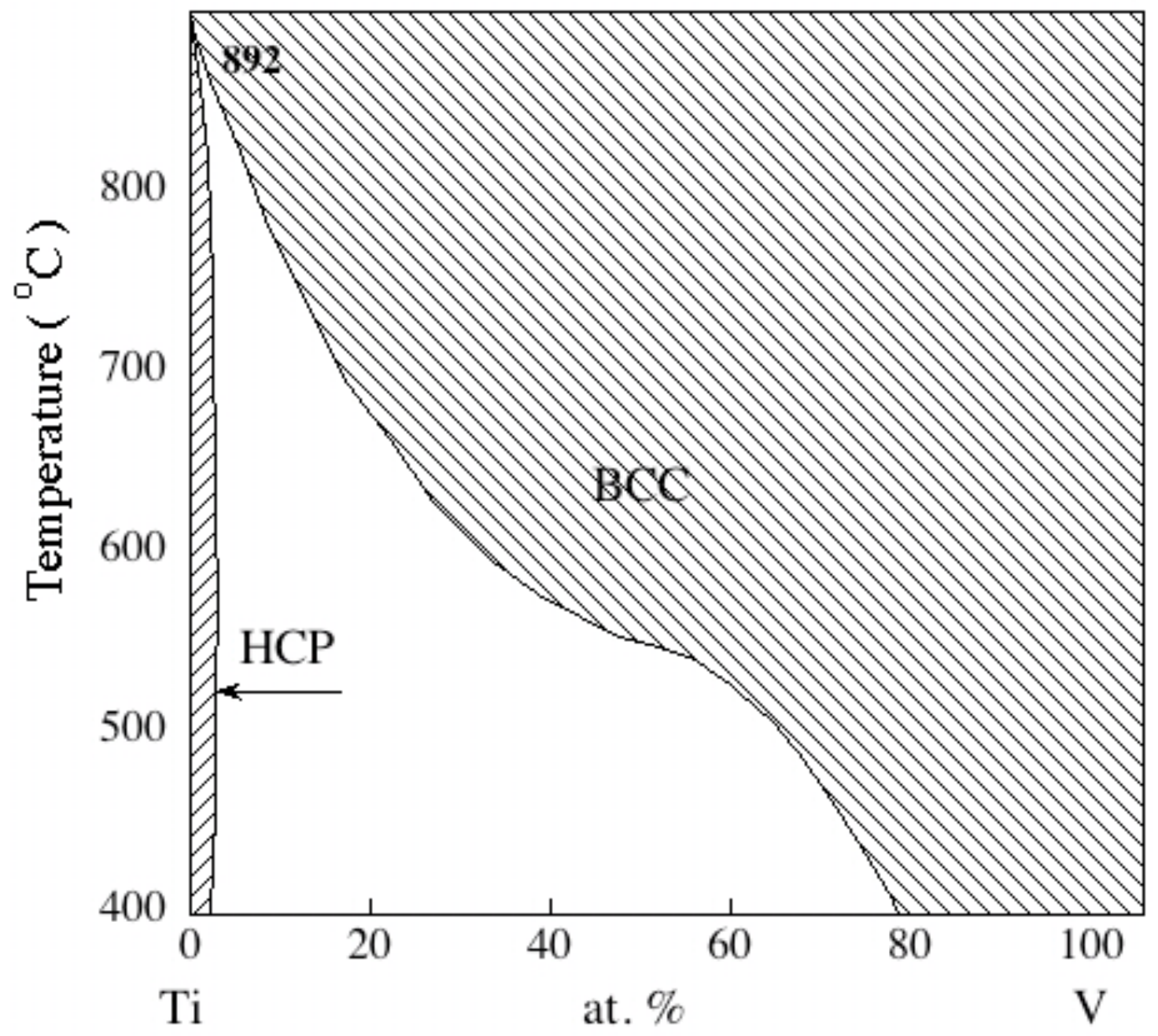


Figure 3

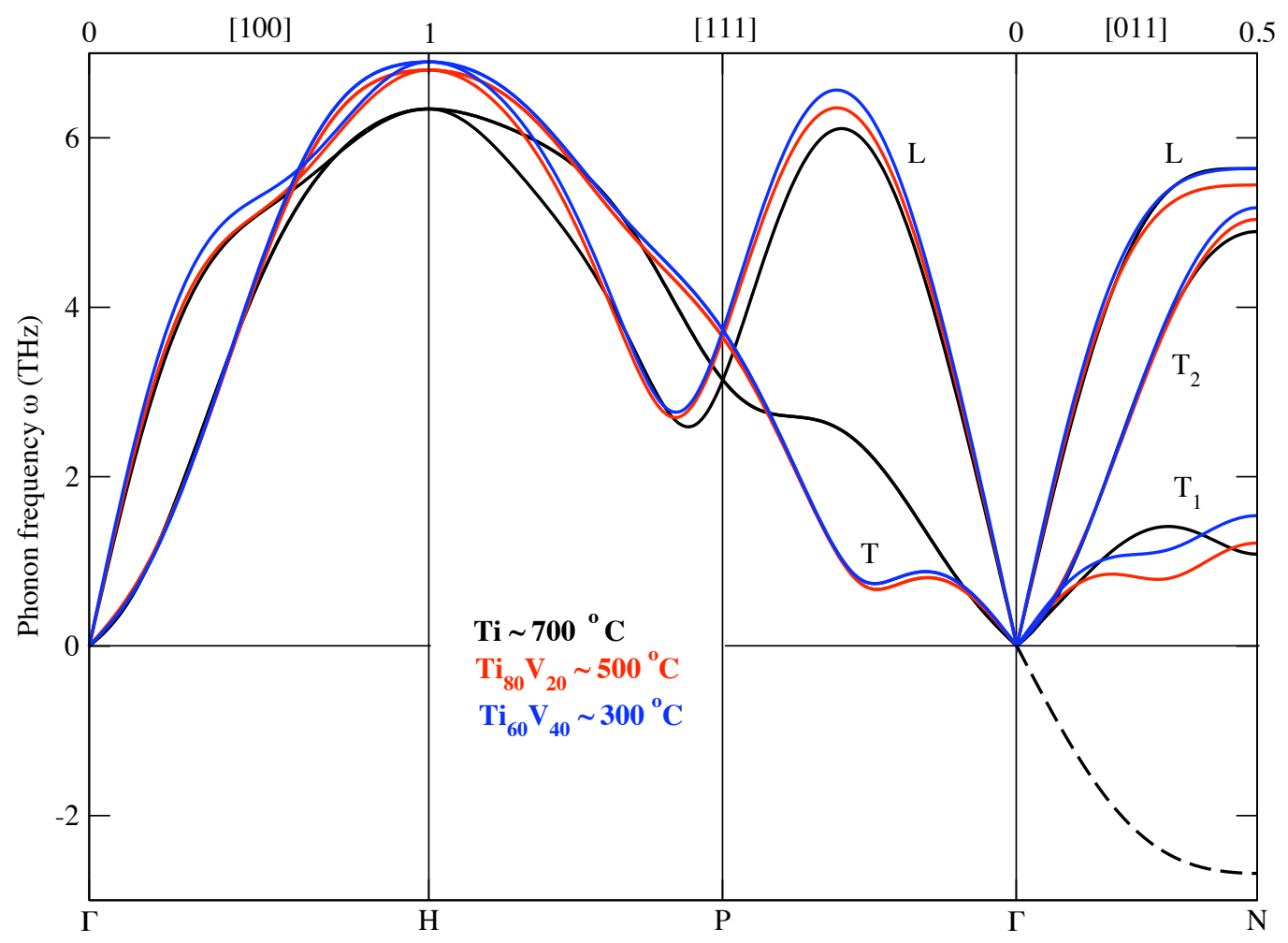


Figure 4

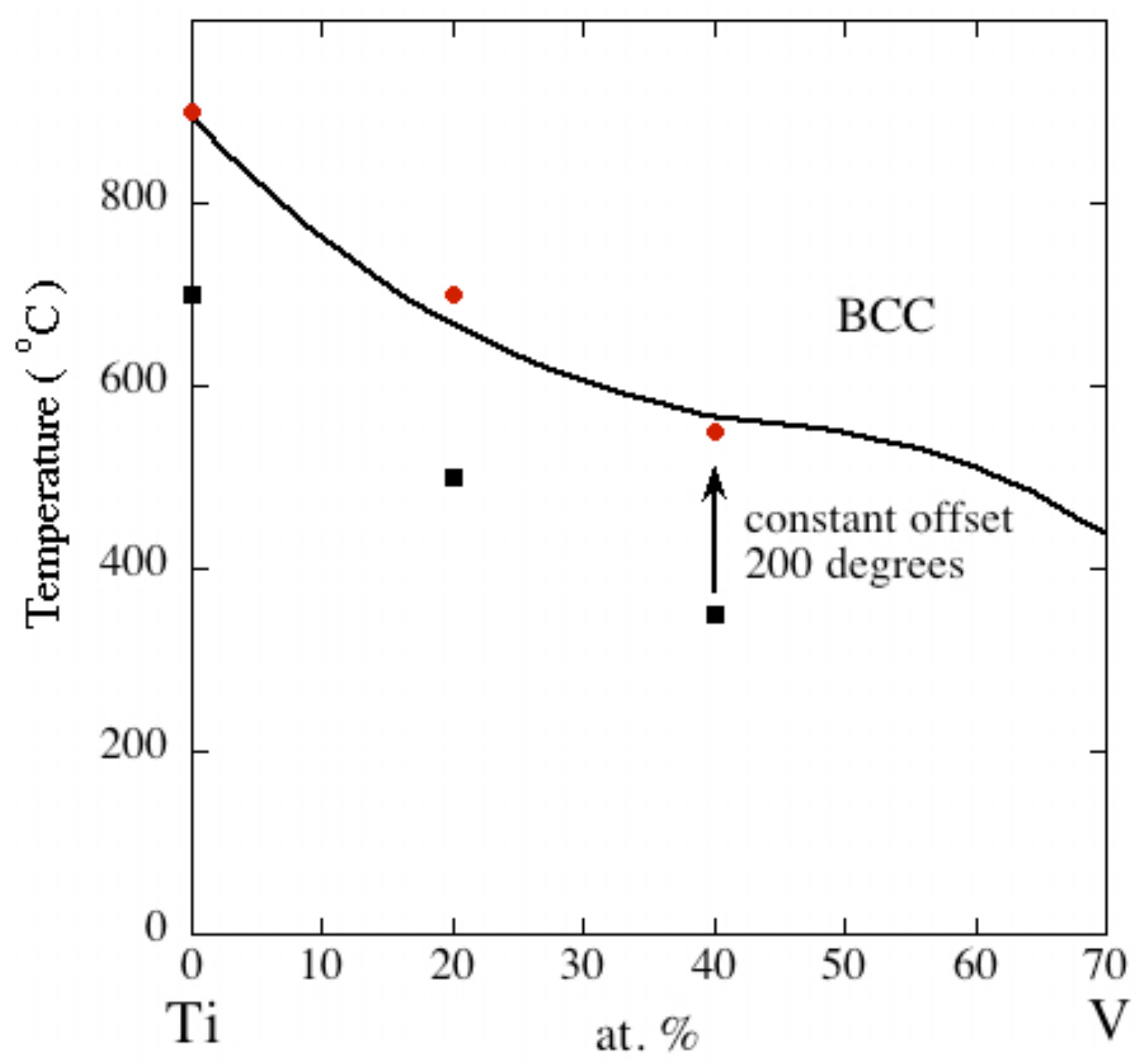


Figure 5

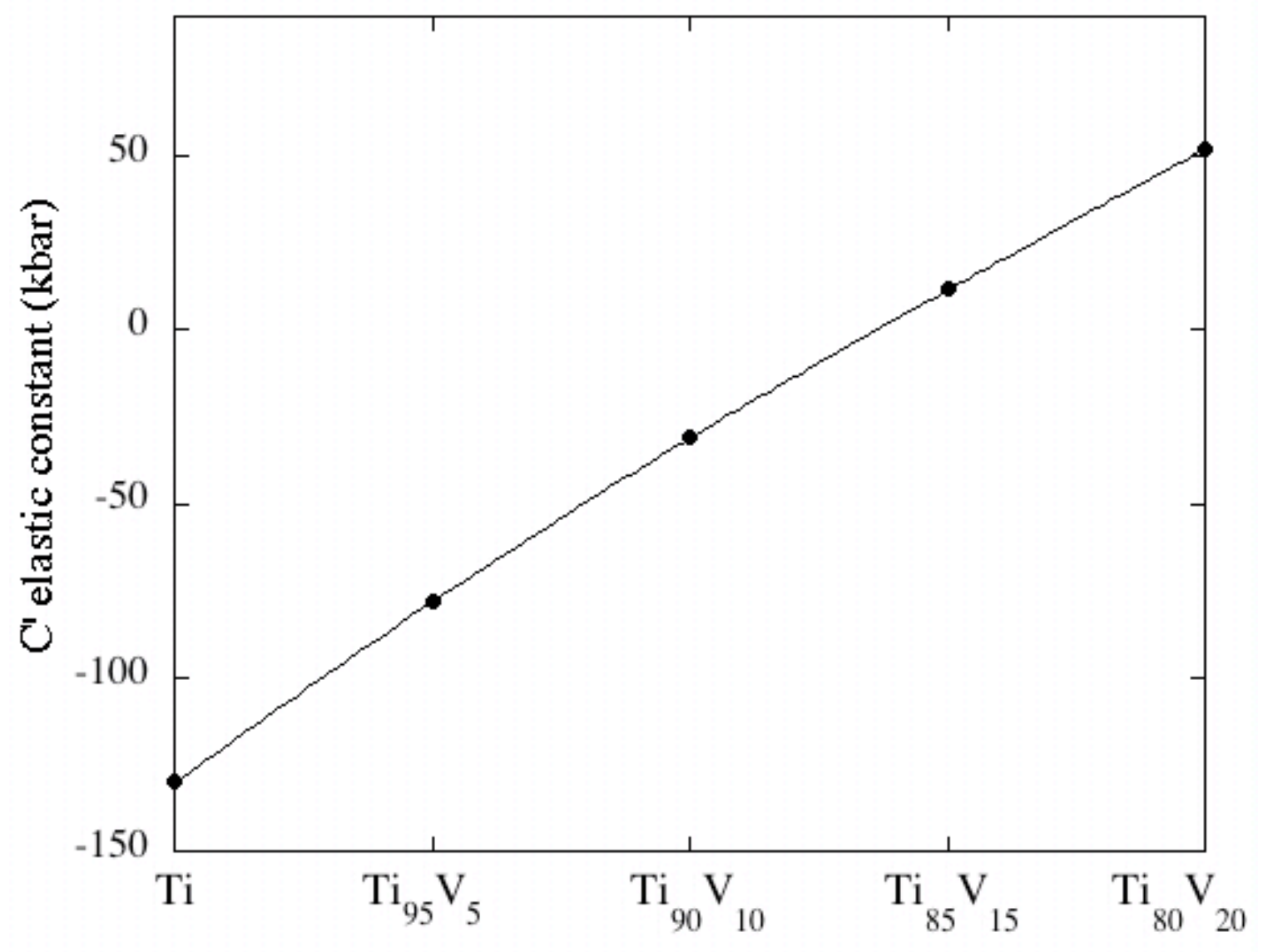

